\title{
Post-COVID-19 Sequelae
}

\author{
Uthara Vijai Kumar \\ Consultant Pulmonologist, Sunshine Group of Hospitals, Hyderabad, Telangana, India
}

\section{Abstract}

COVID-19 is a new disease and the acute clinical presentation is mostly clear now. It is also known now that the disease may have sequelae affecting various systems. The respiratory sequelae include pulmonary fibrosis due to the immune-mediated mechanisms that follow a cytokine storm, diffuse alveolar damage, and microvascular thrombosis. A decline in lung function may be seen in patients who still have residual symptoms and hypoxia. COVID-19-associated pulmonary aspergillosis, a well-recognized complication, especially in patients with acute respiratory distress syndrome, has emerged as a significant risk factor for increased mortality. Fatigue is a common symptom that patients come back with, in the post-COVID period. Dyspnea without hypoxia has been attributed to respiratory muscle dysfunction and deconditioning resulting in decreased exercise tolerance. Palpitation is another common persisting symptom. Thromboembolic disease, a common association during the acute phase of illness, is not an uncommon entity that is seen even after "recovery" from COVID-19. Thromboembolic events causing stroke have been identified as an immediate complication of COVID-19, but can occur during the recovery phase as well, in high-risk patients. The return of smell and taste sensations could take a few weeks to months even after complete recovery from the illness. Mood swings, anxiety, and sleep deprivation have all been reported by patients recovering from this viral illness. The last 14 months have been feverishly spent in trying to understand this particular disease, but the long-term complications of COVID-19 are still elusive.

Keywords: COVID-19-associated pulmonary aspergillosis, post-COVID-19 sequelae, pulmonary fibrosis, thromboembolic disease

\section{INTRODUCTION}

As of February 28, 2021, nearly 113 million people have been infected and 2.5 million people have died from COVID 19 worldwide. In India, close to 11 million people have been infected and 157,000 deaths due to COVID 19 have been reported. ${ }^{[1]}$

The long-term respiratory complications of severe acute respiratory syndrome (SARS) CoV2 infection remain to be seen and further studied. It is a long way to go before we begin to understand the extent and severity of the sequelae caused by the coronavirus although recent data suggest that patients of COVID 19 pneumonia have respiratory symptoms even after weeks or months following their initial illness. ${ }^{[2]}$

When the SARS epidemic ended in June 2003, 8422 people were infected and 916 had died all over the world. The Middle East respiratory syndrome, identified in the year 2012, infected 2519 people and caused 866 deaths worldwide. ${ }^{[3]}$ In patients with SARS, extensive ground-glass opacities with or without consolidations were observed at 2 weeks from symptom onset

\begin{tabular}{|l|l|}
\hline \multicolumn{3}{|c|}{ Access this article online } \\
\hline Quick Response Code: & Website: \\
\hline & www.ijrc.in \\
\hline
\end{tabular}

and $50 \%$ of these patients had reticular changes persisting beyond 4 weeks. ${ }^{[4,5]}$

\section{SequelaE OF COVID-19}

\section{Respiratory}

Pulmonary fibrosis is a well-recognized sequela of acute respiratory distress syndrome (ARDS). Recent data indicate that about $40 \%$ of patients with COVID- 19 admitted to the hospital develop ARDS, and $20 \%$ of them could have severe ARDS. ${ }^{[6]}$

Collective data initially from China and later from Northern Italy confirmed that the most severe disease occurred in elderly men with other poor prognostic factors such as a history of smoking and presence of comorbidities. This demographic is very similar to patients who develop interstitial pulmonary fibrosis. ${ }^{[7]}$

Address for correspondence: Dr. Uthara Vijai Kumar, Sunshine Group of Hospitals, Hyderabad, Telangana, India. E-mail: utharavijai@gmail.com

This is an open access journal, and articles are distributed under the terms of the Creative Commons Attribution-NonCommercial-ShareAlike 4.0 License, which allows others to remix, tweak, and build upon the work non-commercially, as long as appropriate credit is given and the new creations are licensed under the identical terms.

For reprints contact: WKHLRPMedknow_reprints@wolterskluwer.com

How to cite this article: Kumar UV. Post-COVID-19 sequelae. Indian J Respir Care 2021;10:S60-3.

Received: $17-02-2021$

Accepted: 03-03-2021
Revised: 03-03-2021 Published: 29-04-2021 

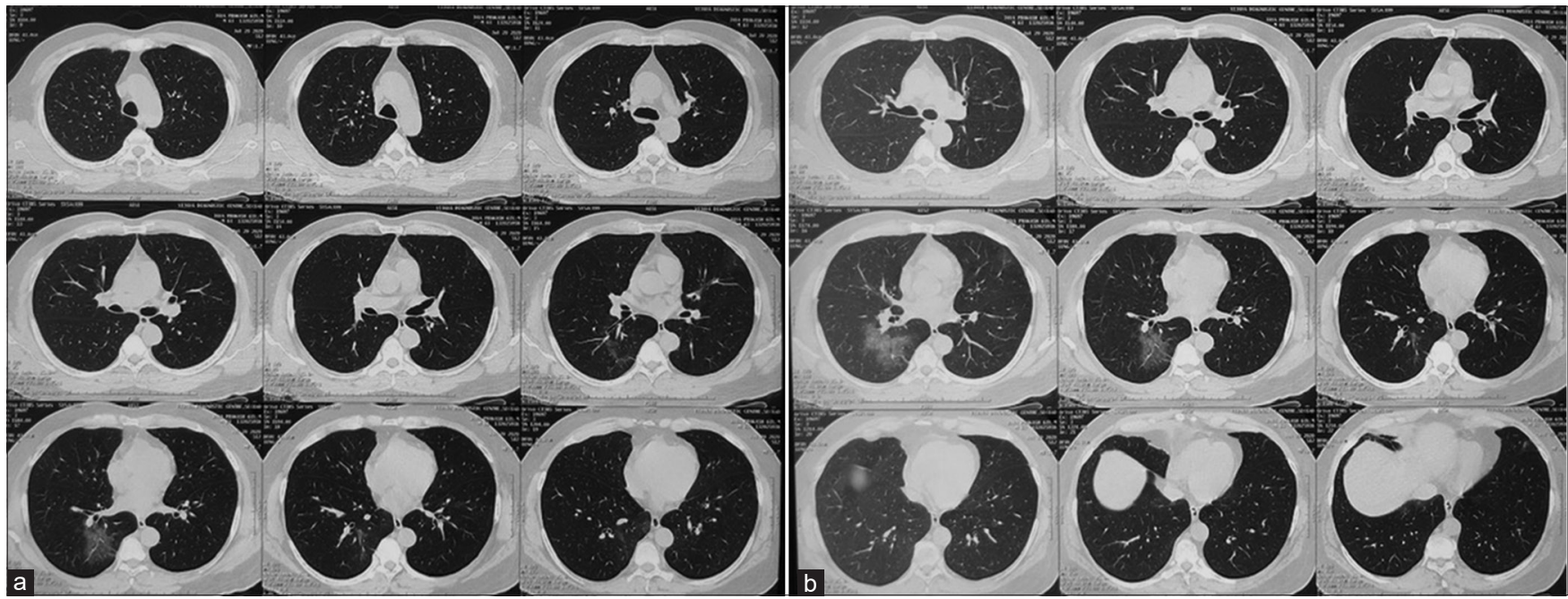

Figure 1: $(\mathrm{a}$ and $\mathrm{b})$ Computerized tomography chest image of a 65-year-old gentleman admitted with COVID-19 pneumonia and respiratory failure showing ground-glass opacities
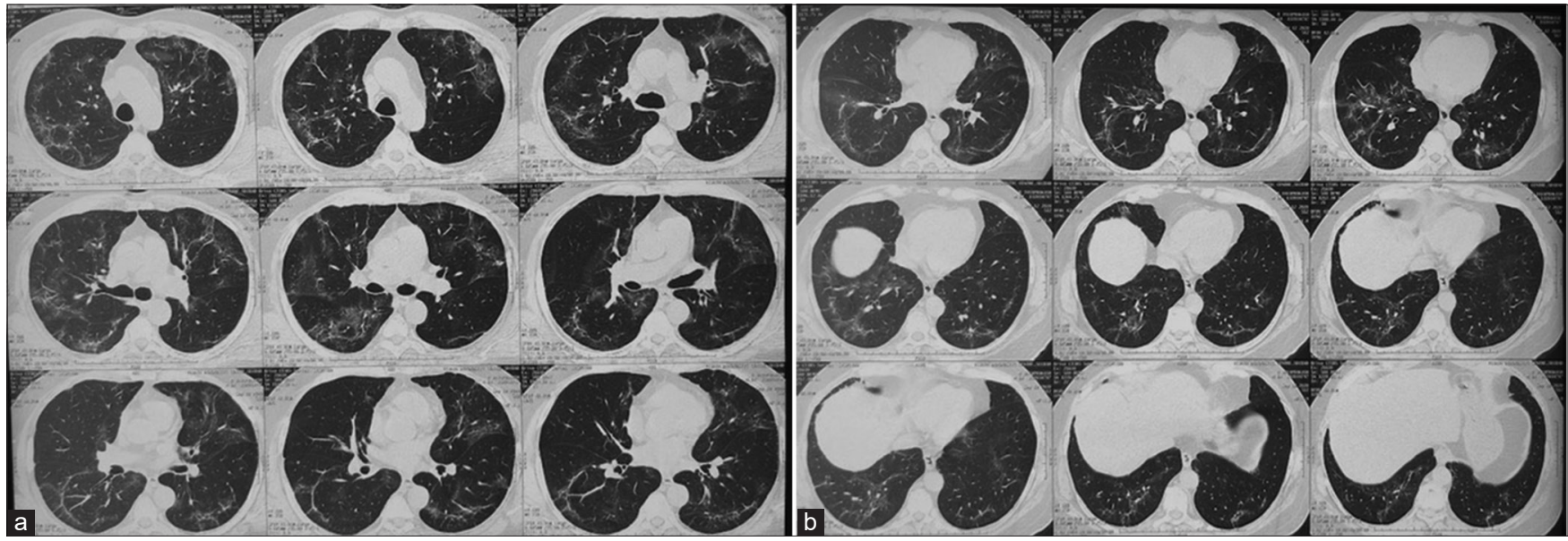

Figure 2: ( $a$ and b) Computerized tomography image of the above-mentioned gentleman after 2 months of tapered steroid therapy which shows early changes of fibrosis

Pulmonary fibrosis in severe SARS CoV2 infection can occur due to the immune-mediated mechanisms that follow a cytokine storm [Figures 1a,b and 2a,b]. Diffuse alveolar damage and microvascular thrombosis on histology have been documented in patients with severe organizing pneumonia and respiratory failure. ${ }^{[7,8]}$ Wang et al. reported in their recent study that $94 \%$ of COVID-19 patients discharged from the hospital had residual disease on their last computerized tomography scans..$^{[9]}$ Now that there is evidence, the use of antifibrotic agents such as pirfenidone and nintedanib is being extrapolated in lung fibrosis resulting from COVID-19 pneumonia, as the burden of fibrotic lung disease world over will increase by significant proportions as an aftermath. ${ }^{[7]}$

A decline in lung function is another cause for concern in patients who have overcome the viral illness but still have residual symptoms and hypoxia. Chen et al. in their pilot study of 51 patients with COVID-19, 29 patients reportedly showed a reduction in both (diffusing capacity of the lung for carbon monoxide (DLCO) and KCO (transfer coefficient of the lung for carbon monoxide) as opposed to 22 patients who only had a reduced DLCO. KCO can be reduced in alveolar-capillary damage, microvascular pathology, or anemia. ${ }^{[10]}$

COVID-19-associated pulmonary aspergillosis (CAPA), a well-recognized complication, especially in patients with ARDS, has emerged as a significant risk factor for increased mortality. Virus-induced damage to the respiratory epithelium resulting in mucociliary dysfunction and dysregulation allows the fungus to invade. The increasing frequency of these infections has been attributed to host factors such as poorly controlled diabetes, use of corticosteroids in severe COVID-19 pneumonia, and anti-interleukin 6 therapy (tocilizumab) in hospitalized patients. Diagnosis of CAPA is based on various host factors, radiological evidence, isolating the fungus in tracheal aspirates or bronchial washings, and serum galactomannan. Voriconazole or 
isavuconazole is the proposed first-line therapy for possible, probable, and proven CAPA. ${ }^{[11]}$

Invasive fungal infections such as invasive candidiasis and mucormycosis have also been reported worldwide as late complications of COVID-19 pneumonia. There was a report from New Delhi where a group of 15 patients with COVID-19 illness eventually developed a bloodstream candida infection. ${ }^{[12]}$

Rhino-orbital-cerebral and pulmonary mucormycosis are two important manifestations of this mold infection in patients undergoing or completed treatment for COVID-19 illness. Biopsy remains the gold standard to isolate the fungus. Initial treatment includes surgical debridement and intravenous amphotericin-B. Oral posaconazole can be used to switch from amphotericin-B to a less nephrotoxic alternative.

\section{Musculoskeletal}

Fatigue is a common symptom that patients come back with, in the post-COVID period. Chronic fatigue or myalgic encephalomyelitis has been previously described in other viral diseases in the past.

A recent Italian study of 143 post-COVID-19 patients showed that $87.4 \%$ of patients had at least one symptom persisting even after recovery from the acute phase. Fatigue was reported by $53 \%$ followed by dyspnea (43\%), joint pain $(27.3 \%)$, and chest pain $(21.7 \%){ }^{[13]}$

Dyspnea without hypoxia has been attributed to respiratory muscle dysfunction and deconditioning resulting in decreased exercise tolerance. Cardiopulmonary exercise testing could be a good diagnostic tool in patients who have exertional dyspnea despite normal lung volumes and cardiac function.

\section{Cardiovascular}

Thromboembolic disease has been described as a common association during the acute phase of illness but is not an uncommon entity that is seen even after "recovery" from COVID-19. A study by Ackermann et al. suggested that the COVID-19 lung showed features of endothelial cell dysfunction consistent with vasculitis. ${ }^{[14]}$

Palpitation is another common persisting symptom. In an observational study in which post-COVID patients underwent cardiovascular magnetic resonance imaging 70 days after the initial diagnosis, 78 out of 100 patients had abnormal findings. ${ }^{[15]}$

\section{Neuropsychiatric}

Loss of sense of smell and taste has been one of the cardinal symptoms of COVID-19, but in some adults, the return of these sensations to normal could take a few weeks to months even after complete recovery from the illness.

Thromboembolic events causing stroke have been identified as an immediate complication of COVID-19, but can occur during the recovery phase as well, in high-risk patients.

The COVID-19 pandemic has had a devastating psychosocial impact on the society. The most vulnerable groups have been children, the elderly, frontline health-care workers, people with pre-existing mental illness, COVID-19 survivors, and families in bereavement. ${ }^{[16]}$

Mood swings, anxiety, and sleep deprivation have all been reported by patients recovering from this viral illness. ${ }^{[17]}$

As convalescent patients come to the outpatient clinic seeking answers for their persisting symptoms, more studies are needed to ascertain whether this viral illness is associated with metabolic conditions such as diabetes and how long it will take for patients to get back to their regular health trajectory.

To conclude, the last 14 months have been feverishly spent in trying to understand this particular disease, but the long-term complications of COVID-19 are still elusive. It is time we identified the dire consequences this illness can have on the health of the general population and create a support system which is needed to overcome this devastating pandemic.

\section{Declaration of patient consent}

The authors certify that they have obtained all appropriate patient consent forms. In the form the patient(s) has/have given his/her/their consent for his/her/their images and other clinical information to be reported in the journal. The patients understand that their names and initials will not be published and due efforts will be made to conceal their identity, but anonymity cannot be guaranteed.

\section{Financial support and sponsorship}

Nil.

\section{Conflicts of interest}

There are no conflicts of interest.

\section{RefERENCES}

1. WHO. Coronavirus Disease 2019(COVID-19) Situation Report. Available from: https://www. who.int/emergencies/diseases/novelcoronavirus-2019/events-as-they-happen. [Last accessed on 2021 Feb 28].

2. Fraser E. Long term respiratory complications of covid-19 BMJ 2020; 370:m3001.

3. National Institute of Allergy and Infectious Diseases. COVID-19, MERS \& SARS; 2020. Available from: https://www.niaid.nih.gov/ diseases-conditions/COVID-19. [Last accessed on $2021 \mathrm{Feb} 28$ ].

4. Ooi GC, Khong PL, Müller NL, Yiu WC, Zhou LJ, Ho JC, et al. Severe acute respiratory syndrome: Temporal lung changes at thin-section CT in 30 patients. Radiology 2004;230:836-44.

5. Zhang P, Li J, Liu H, Han N, Ju J, Kou Y, et al. Long-term bone and lung consequences associated with hospital-acquired severe acute respiratory syndrome: A 15-year follow-up from a prospective cohort study. Bone Res 2020;8:8.

6. Wu C, Chen X, Cai Y, Xia J, Zhou X, Xu S, et al. Risk factors associated with acute respiratory distress syndrome and death in patients with coronavirus disease 2019 pneumonia in Wuhan, China. JAMA Intern Med 2020;180:934-43.

7. George PM, Wells AU, Jenkins RG. Pulmonary fibrosis and COVID-19: The potential role for antifibrotic therapy. Lancet Respir Med 2020;8:807-15.

8. Zhang T, Sun LX, Feng RE. Comparison of clinical and pathological features between severe acute respiratory syndrome and coronavirus disease 2019. Zhonghua Jie He He Hu Xi Za Zhi 2020;43:496-502.

9. Wang Y, Dong C, Hu Y, Li C, Ren Q, Zhang X, et al. Temporal changes of CT findings in 90 patients with COVID-19 pneumonia: A longitudinal study. Radiology 2020;296:E55-64. 
10. Chen R, Gao Y, Chen M, Jian W, Lei C, Zheng J, et al. Impaired pulmonary function in discharged patients with COVID-19: More work ahead. Eur Respir J 2020;56:2002194.

11. Koehler P, Bassetti M, Chakrabarti A, Chen SCA, Colombo AL, Hoenigl $\mathrm{M}$, et al. Defining and managing COVID-19-associated pulmonary aspergillosis: The 2020 ECMM/ISHAM consensus criteria for research and clinical guidance. Lancet Infect Dis 2020;S1473-3099(20)30847-1.

12. Chowdhary A, Tarai B, Singh A, Sharma A. Multidrug-resistant candida auris infections in critically Ill coronavirus disease patients, India, April-July 2020. Emerg Infect Dis 2020;26:2694-6.

13. Carfi A, Bernabei R, Landi F; Gemelli Against COVID-19 Post-Acute Care Study Group. Persistent symptoms in patients after acute COVID-19. JAMA 2020;324:603-5.

14. Ackermann M, Verleden SE, Kuehnel M, Haverich A, Welte T,
Laenger F, et al. Pulmonary vascular endothelialitis, thrombosis, and angiogenesis in Covid-19. N Engl J Med 2020;383:120-8.

15. Puntmann VO, Carerj ML, Wieters I, Fahim M, Arendt C, Hoffmann J, et al. Outcomes of cardiovascular magnetic resonance imaging in patients recently recovered from coronavirus disease 2019 (COVID-19). JAMA Cardiol 2020;5:1265-73.

16. Holmes EA, O'Connor RC, Perry VH, Tracey I, Wessely S, Arseneault L, et al. Multidisciplinary research priorities for the COVID-19 pandemic: A call for action for mental health science. Lancet Psychiatry 2020;7:547-60.

17. Garner P. COVID-19 at 14 Weeks-Phantom Speed Cameras, Unknown Limits, and Harsh Penalties; 2020. Available from: https://blogs.bmj. $\mathrm{com} / \mathrm{bmj} / 2020 / 06 / 23 /$ paul-garner-COVID-19-at-14-weeks-phantomspeed-cameras-unknownlimits-and-harsh-penalties/.[Last accessed on 2020 Sep 29]. 